\title{
USING COMPUTER GRAPHICS TO DESIGN SPACE STATION FREEDOM VIEWING
}

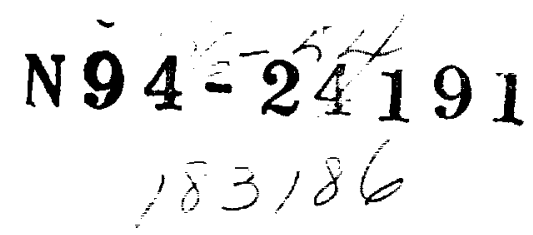

\author{
Betty S. Goldsberry, Buddy O. Lippert, and Sandra D. McKee \\ Lockheed Engineering and Sciences Company \\ James L. Lewis, Jr. and Francis E. Mount \\ NASA Lyndon B. Johnson Space Center
}

\section{INTRODUCTION}

Viewing requirements were identified early in the Space Station Freedom program for both direct viewing via windows and indirect viewing via cameras and closed-circuit television (CCTV). These requirements reside in NASA Program Definition and Requirements Document (PDRD), Section 3: Space Station Systems Requirements.

Currently, analyses are addressing the feasibility of direct and indirect viewing. The goal of these analyses is to determine the optimum locations for the windows, cameras, and CCTVs in order to meet established requirements, to adequately support space station assembly, and to operate on-board equipment.

PLAID, a three-dimensional computer graphics program developed at NASA JSC, was selected for use as the major tool in these analyses. PLAID provides the capability to simulate the assembly of the station, as well as to examine operations as the station evolves. This program has been used successfully as a tool to analyze general viewing conditions for many Space Shuttle elements and can be used for virtually all Space Station components. Additionally, PLAID provides the ability to integrate an anthropometric scale-modeled human (representing a crewmember) with interior and exterior architecture.

\section{BACKGROUND}

\section{COMPUTER SIMULATION}

The design of a computer simulation system, such as PLAID, that includes human models is a complex process. Total system performance is dependent on the accuracy of the models generated as well as the interactions between humans, hardware, and software. Model requirements can be based on workspace geometry, figure anthropometry, strength/force characteristics, and reach envelopes. If results of simulation analyses are to be valuable, development of the models must be based on a valid representation of the environment. Also, procedures must be employed that make available alternative human/system designs. Ultimately, quantitative predictions of events and behaviors in response to realistic operating conditions for various design alternatives must be compared in order to select the optimum design characteristics.

While there are many ways of quantitatively predicting human behavior and performance, computer simulation of the human operator in a mission context represents a method that is internally consistent and compatible with other contemporary system engineering evaluative techniques. Computer simulations usually resolve many design and development problems in an effective manner earlier than experiments with human subjects. Even though simulation does not eliminate the need for empirical tests, properly exploited, it can help to focus test time and energy on appropriate issues and potential problems.

The analyst interested in evaluating human performance can use computer simulation to construct a graphic human model in much the same way that an airplane or automobile designer can model a vehicle. Then, the model can be used to test various design concepts relevant to human-system interactions and integrations. Actually, a computer simulation 
system can be developed long before a prototype or test facility can be made operational.

\section{PLAID MAN-MODELING SYSTEM}

PLAID uses anthropometric data collected from astronaut candidates in the JSC Anthropometrics and Biomechanics Laboratory to generate human models with realistic joint limits and user-specified size characteristics. A high-fidelity model of the Space Shuttle Remote Manipulator System (RMS) is also contained in the PLAID database. For many years, the models have facilitated the comparison of reach envelopes for humans and remote manipulators in order to determine which space operations are feasible for astronaut extravehicular activities (EVA) and which are more appropriately accomplished by robotics applications. PLAID has also played a major role in satellite retrieval execution, equipment failure diagnosis, and vehicle damage prediction.

PLAID is a three-dimensional-solids modeling system which generates computer models that can be examined from an infinite number of viewpoints. This feature allows the system user to position its viewpoint where the eyes of the crewmember or lens of the camera would be located. Then, subsequent analyses can reveal what a crewmember can or cannot see. The PLAID system has taken into consideration that the visual environment of space is different from a normal Earth atmosphere and has incorporated appropriate contrast ratios, shadowing, and light scattering for the space environment.

\section{SPACE STATION \\ PROCEDURE}

Since viewing tasks vary in complexity, relevant viewing requirements and proposed window locations were identified. Using the PLAID system, geometric models of Space Station Freedom elements and configuration models were created. These models reflected up-to-date Space Station Freedom architectural information for internal and external elements.
Also, proposed window and camera locations were integrated with the models. Then, computer graphics of various viewing scenarios were generated which revealed fields-of-view for selected locations of interest. Finally, the graphics generated by the system were analyzed relative to published design requirements and specifications.

The simulation is influenced by more than a three-dimensional layout of the environment. In particular, task sequence and task time are important ingredients. For example, if the humans must monitor exterior facilities while operating interior controls, their fields-of-view will be decreased because their positions will probably be farther from the window. Shadowing can also reduce visibility.

The simulation helped analysts evaluate the proposed window locations and identify problem areas. Recommended window placements were determined by the integration of the view available from a particular position with various other factors such as operations requirements, anthropometric clearances, traffic flow, and window accessibility.

\section{DATA}

Data in the form of PLAID graphics were generated and maintained at the JSC/ManSystems Division. Figures 1 through 4 , illustrated on the following page, exemplify the types of data used in the analyses. Figure 1 was generated to determine the viewing clearance needed if an airlock were placed near the window.

Figure 2 illustrates that the cupola will accommodate two crewmembers. Note the design requirement that the Crew Escape Rescue Vehicle (CERV) be visible from the cupola is met. Displays that share the crewmembers' fields-of-view, are shown.

Figures 3 and 4 illustrate that viewing of the external environment can be accomplished. Analyses have determined that Earth viewing or optic experiments could be obstructed by truss work from some locations. 


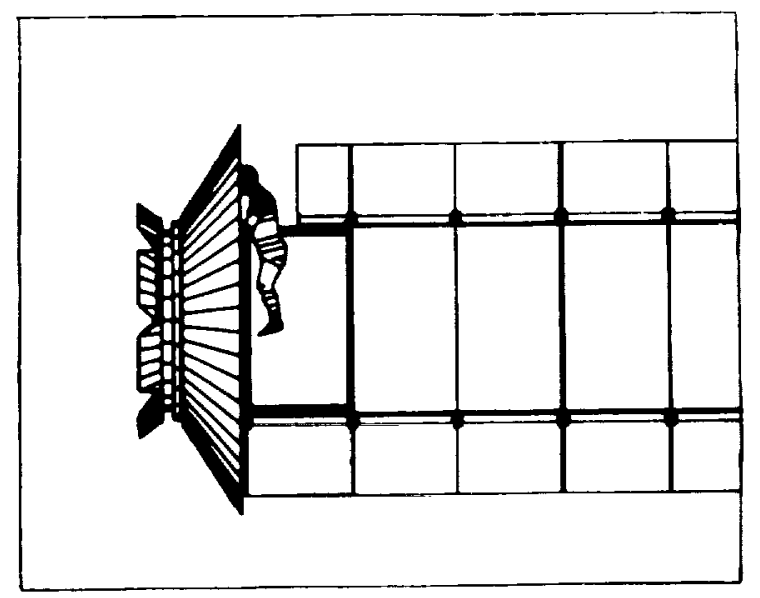

Figure 1. A 95th percentile male crewmember looking out of the cone-shaped end portion of a module (endcone) window.

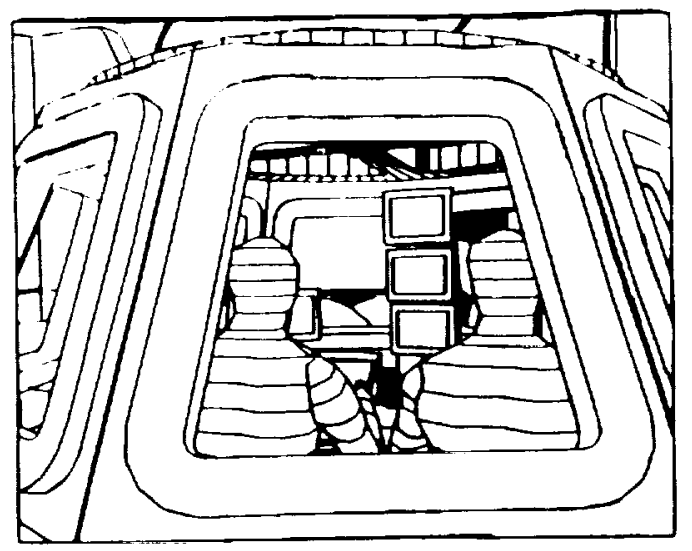

Figure 2. Two 50th percentile males in a cupola.

\section{DOCUMENTATION}

Comprehensive documentation of the Space Station Viewing Analyses results will be organized in a series of volumes. Volume 1 covers viewing of the Space Station Freedom assembly sequence as seen through the Orbiter windows and CCTVs. Volume 2 uses the Man-Systems candidate topologies to evaluate possible window locations in the U.S. Habitation module. Volume 3 evaluates the candidate window locations as proposed by the

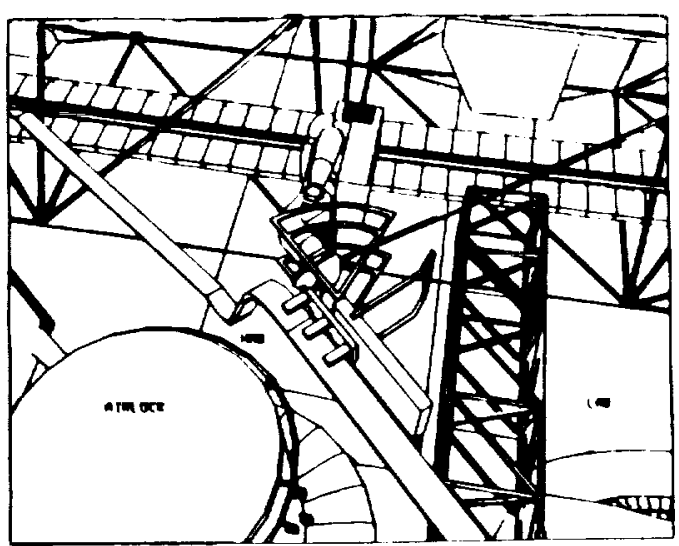

Figure 3. A view of extravehicular activity (EVA).

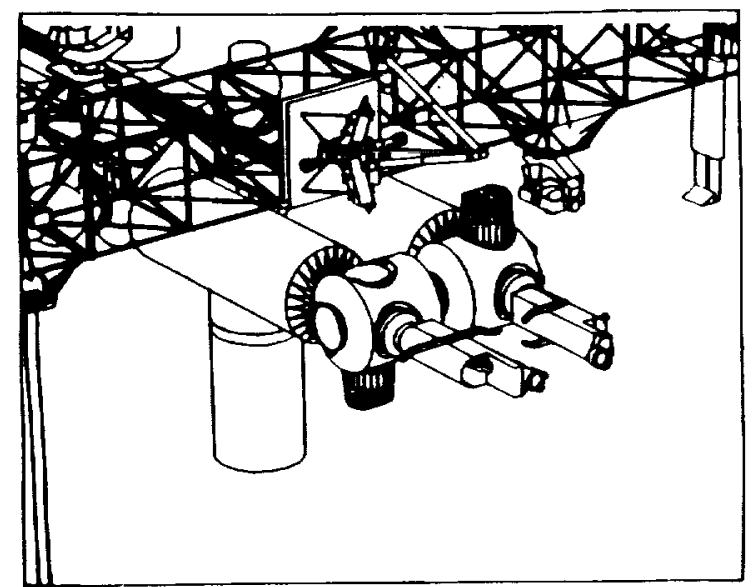

Figure 4. An Orbiter window or external camera view during approach or departure.

European Space Agency (ESA) for the Columbus Module, as well as selected alternate locations. Volume 4 addresses viewing conditions of the National Space Development Agency (NASDA) Japanese Experiment Module (JEM). Volume 5 is dedicated to the discussion of viewing from the nodes and the two cupolas baselined on nodes three and four. Volume 6 addresses indirect viewing and the placement of cameras throughout the station. Volume 7 pertains to the viewing conditions of the U.S. Laboratory module. Volume 8 will 
address Phase II Space Station Freedom viewing impacts. The planned order of preparation for these basic volumes is $3,2,4$, $7,5,1,6$, and 8 . The first four volumes in this series have been released. Published recommendations from the completed volumes follow.

\section{RECOMMENDATIONS}

\section{U. S. Habitation Module}

1. A requirement for windows in the crew quarters should be established by the program.

2. Two viewing stations, currently proposed, should be relocated to positions that will provide unobstructed views.

3. Modular interior layout and wardroom area window placement should be integrated such that recreational viewing (i.e., viewing of the Earth limb) is optimized. Selected port rack locations are well suited for wardroom area windows.

4. Windows should be placed in the portion of the quadrant where the eyes are most likely to be, while maintaining compliance with the local vertical.

\section{U. S. Laboratory Module}

1. Evaluate scientific celestial viewing from the zenith $(-Z)$ oriented windows in the module to insure minimal interference of Space Station elements.

2. A proposed floor window is suitable for Earth viewing.

3. Optimum high-oblique viewing in the $-Y$ direction for scientific uses can be achieved from a starboard window. Exact placement of the window below the module centerline should be determined based upon intended use of the window.

\section{ESA Columbus Module (CM)}

1. Design of the module should provide ample space for movement, interactions, interfaces, and other human factors considerations.

2. Orient a minimum of two windows in the $\mathrm{CM}$ in the $+\mathrm{X}$ and $-\mathrm{X}$ directions.

3. Rotate the aft endcone at least 45 degrees from 12 o'clock position to meet Space Station Program 30000 requirements and support recreational viewing.

4. Rotate the window location in the forward endcone of the CM 45 degrees clockwise.

5. Consider the need for celestial viewing from the $\mathrm{CM}$ in the overall station configuration.

6. Establish commonality of window design characteristics in all modules.

7. Supplement the view through the hatch viewport by providing interior video which could also be used to remotely monitor activities within the modules.

\section{NASDA Japanese Experiment Module}

1. Supplement direct viewing of Mobile Servicing Center (MSC) operations by using the CCTV for indirect viewing.

2. Supplement direct viewing of the Experiment Logistics Module (ELM) transfer and berthing/deberthing operation by using the CCTV for indirect viewing and for supporting direct viewing from the hatch viewport beneath the JEM berthing ring during the berthing process.

3. Retain proposed windows in the aft bulkhead.

4. Retain baseline with no windows in the forward bulkhead, since no meaningful view could be achieved.

5. Retain floor window for Earth observation.

6. For purposes of celestial viewing, use the viewport between the JEM and ELM during absence of the ELM. Further information is 
needed concerning (a) duration of the ELM's absence and (b) external covering of the viewpoint during ELM's absence.

\section{CONCLUSION}

\section{VIEWING ANALYSIS}

PLAID allows for an in-depth analysis of the impact of direct and indirect viewing on architectural design and human performance issues. Specifically, window and video location selections have been facilitated by the use of this computer simulation system. This system permits the integration of selected window/camera locations with the internal architecture, the validation of crew activity sequences, and the determination of necessary body movements required to accomplish viewing tasks. Through findings in these analyses, analysts have been able to write a Change Request (CR) affecting high level Space Station Program documents. Requests address placement issues in the modules. Future volumes will address indirect viewing, cupola viewing, node windows, and Space Station assembly.

\section{PLAID SYSTEM}

Development of a more interactive, real-time system which uses natural language will enhance capabilities of the system. Expansion of the human-modeling capability will include a population range from the 95 th percentile to the 5th percentile female. Currently, work in this area is being conducted for JSC and other users at the University of Pennsylvania under the direction of Norman Badler, PhD.

Also, the capability of generating video animation sequences is being added to PLAID. This feature will allow for simulation of a complete task sequence rather than a "snapshot" approach. Animation will provide for a more comprehensive evaluation of crewmember activities, as well as reveal the relationship between a series of contiguous activities. By following the action flow, the system user can isolate spatial interferences, procedural inconsistencies, and crewmember interactions.
Finally, a dynamic program extension is planned that will allow system users to more accurately evaluate factors such as force and path trajectory. In addition, an efficient ray trace algorithm would enhance lighting study capabilities.

\section{ACKNOWLEDGEMENTS}

These viewing analyses could not have been accomplished without the NASA Graphics Analysis Facility, Facility Manager Linda Orr, and Lockheed technical support.

\section{REFERENCES}

1. Mount, Frances E. and McKee, Sandra. Space Station Freedom Viewing Analysis: Volumes 2, 3, 4, and 7, JSC No. 32089, NASA Johnson Space Center, 1989.

2. Mount, Frances E. and Lewis, James L. Space Station Viewing Requirements (1986). 1986 SAE Aerospace Technology Conference and Exposition. 